\title{
Structural Effects in the Protonic/Electronic Conductivity of Dion-Jacobson Phase Niobate and Tantalate Layered Perovskites Supporting Information
}

\author{
Yoji Kobayashi, Joshua A. Schottenfeld, Digby D. Macdonald, Thomas E. Mallouk.
}

104 Chemistry Building, University Park, PA 16801

\section{Figures S1-S3}

XRD shows that the tantalate analogues of the $\mathrm{HLaNb}_{2} \mathrm{O}_{7}$ all have similar structure and somewhat similar dehydration temperatures. Figure $\mathbf{S 3}$ visually shows the effect of water vapor on suppressing the reduction of $\mathrm{HLaNb}_{2} \mathrm{O}_{7}$. Small amounts of the material were placed in a quartz tube and heated in tube furnace, and pictures were taken as the temperature was ramped upwards. The blue color indicates that the material has been reduced extensively under dry hydrogen.

\section{Figures S4-S5 (Kramers-Kronig Transforms)}

Kramers-Kronig transforms were conducted on select data to test for stability of the system during measurement. The transforms are a purely mathematic way of testing for conformity to Linear Systems Theory, ${ }^{1,2}$ or causality, linearity, and stability. The transforms were conducted by a custom-written $\mathrm{C}++$ code. ${ }^{3}$ In Figure S4, the experimental results were fitted with a polynomial before performing integration, and hence some low and high frequency data points have been omitted on the graph due to inaccuracies in integrating the tails. In Figure S5, an equivalent circuit was fitted to data which permitted the whole measure frequency range to be fitted. Both figures show close agreement between fitted and measured values, implying that the system was indeed stable during measurement.

\footnotetext{
1 R. L. Kronig, J. Opt. Ann., 1926, 12, 547

2 Kramers, H. A. Z. Phys., 1929, 30, 521.

${ }^{3}$ Written by M. Urquidi-Maconald and D. D. Macdonald, 2001.
} 


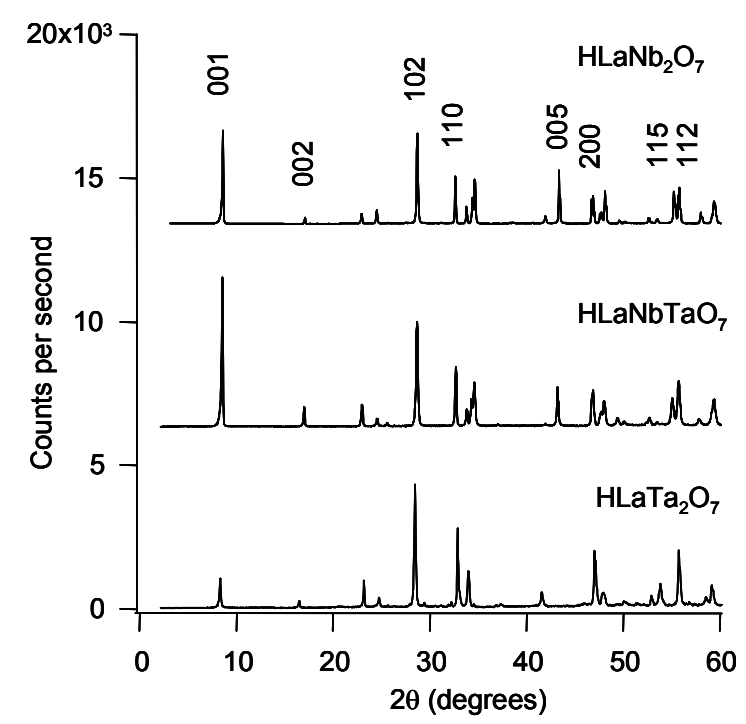

Figure S1. X-ray diffraction patterns of B-site $\mathrm{HLaNb}_{2} \mathrm{O}_{7}$ analogues.

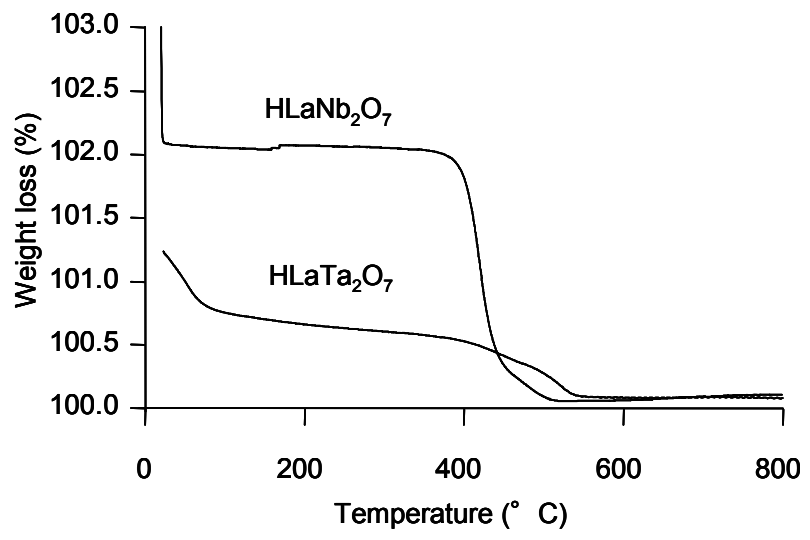

Figure S2. Thermal gravimetry of $\mathrm{HLaNb}_{2} \mathrm{O}_{7}$ and $\mathrm{HLaTa}_{2} \mathrm{O}_{7}\left(1^{\circ} \mathrm{C} / \mathrm{min}\right)$. Sample equilibrated at $100 \% \mathrm{RH}$ prior to analysis. 


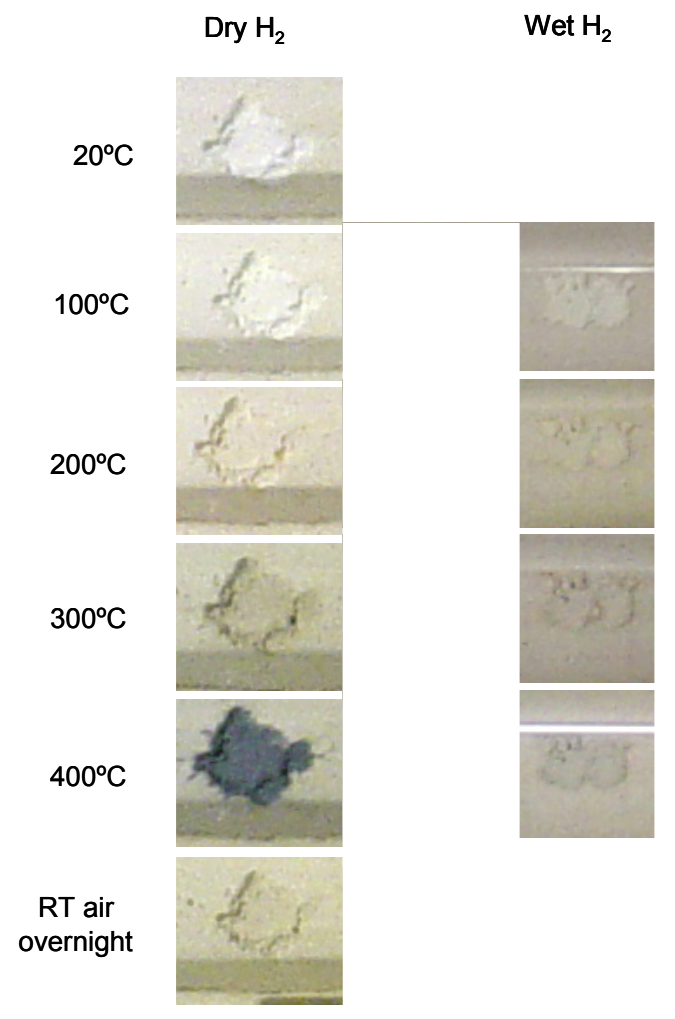

Figure S3. Reduction of $\mathrm{HLaNb}_{2} \mathrm{O}_{7}$ under dry/ wet hydrogen $\left(\mathrm{P}_{\mathrm{H} 2 \mathrm{O}}=0.3 \mathrm{~atm}\right)$. 

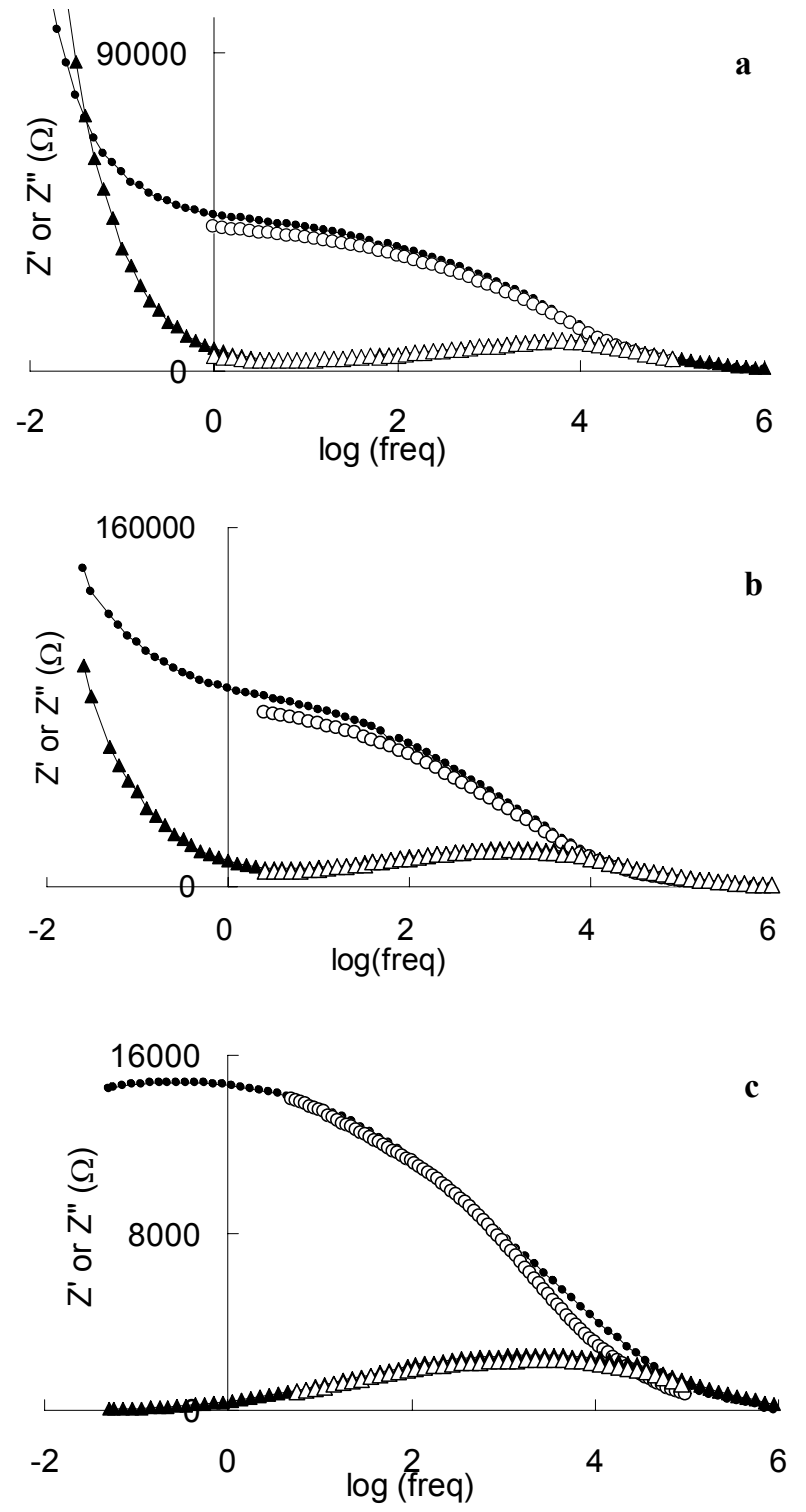

Figure S4. Kramers-Kronig transforms of impedance spectra shown in various figures of the main text.

(a): open squares from Fig. 1

(b): open squares from Fig. 4a

(c): closed circles from Fig. 4a.

: Z" from experiment

O: Z" from KK transform

$\Delta$ : $Z$ ' from experiment

$\Delta: \quad Z^{\prime}$ from $\mathrm{KK}$ transform 

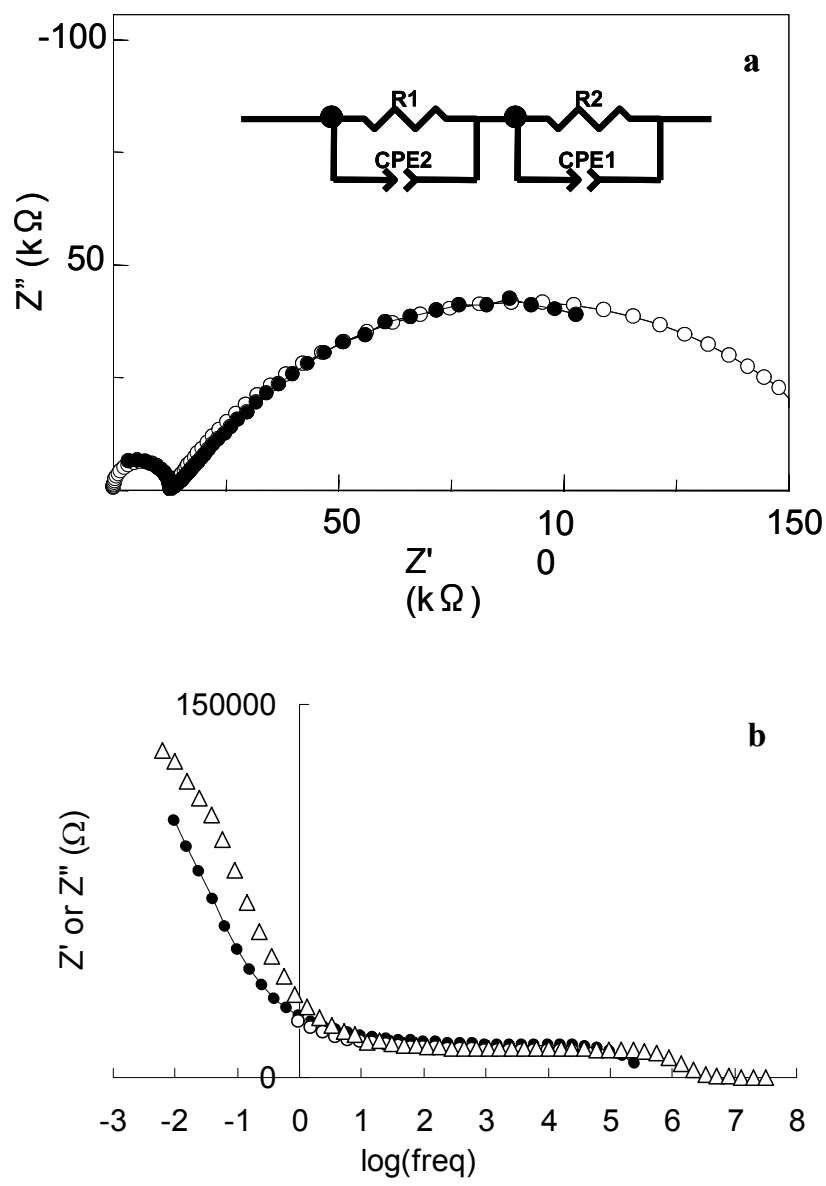

Figure S5. Kramers-Kronig transforms of impedance spectra shown in Fig. 1, using a model circuit to treat the tails of the measured region.

(a): : experimental data

O: fitted data

(b): : experimental data

O: KK transform conducted without fitted data

$\triangle:$ KK transform conducted with fitted data 